\title{
Should my composite restorations last forever? Why are they failing?
}

\section{Flávio Fernando DEMARCO(a) Kavê COLLARES(a) \\ Marcos Britto CORREA(a) Maximiliano Sergio CENCI(a) Rafael Ratto de MORAES(a) Niek Johannes OPDAM(b)}

(a) Universidade Federal de Pelotas - Unipel, School of Dentistry, Graduate Program in Dentistry, Pelotas, RS, Brazil.

(b) Radboud University Medical Center, Radboud Institute for Health Sciences, Department of Dentistry, Niimegen, The Netherlands.
Declaration of Interests: The authors certify that they have no commercial or associative interest that represents a conflict of interest in connection with the manuscript.

\section{Corresponding Author:}

Flávio Fernando Demarco

E-mail: flavio.demarco@ufpel.edu.br

https://doi.org/10.1590/1807-3107BOR-2017.vol31.0056

Submitted: May 12, 2017

Accepted for publication: May 22, 2017

Last revision: May 24, 2017
Abstract: Composites resins have become the first choice for direct anterior and posterior restorations. The great popularity is related to their esthetic appearance and reduced need of sound tissue removal as compared with former treatments. Several studies have demonstrated that composite restorations may last long in clinical service. In this review we discuss the factors playing a role on the long-term longevity. Composite restorations have demonstrated a good clinical performance with annual failure rates varying from $1 \%$ to $3 \%$ in posterior teeth and $1 \%$ to $5 \%$ in anterior teeth. Factors related to the patients such as caries risk and occlusal stress risk, in addition to socioeconomic factors, may affect the survival significantly. Characteristics of the clinical operators, particularly their decision making when it comes to observing or approaching an existing restoration, are decisive for longevity. Cavity features such as the number of restored walls, composite volume, and presence of endodontic treatment are of major importance and may dictate the service time of the restorative approach. The choice of restorative composite seems to have a minor effect on longevity provided that appropriate technical procedures are used. The main reasons for failure in posterior teeth are secondary caries and fracture (restoration or tooth/restoration), while in anterior teeth esthetic concerns are the main reasons leading to restoration failures. Composite resin restorations can be considered a reliable treatment as long as both the professional and the patient are aware of the factors involved in restoration failures.

Keywords: Clinical Study; Longevity; Composite Resins; Dental Restoration

\section{Introduction}

Even though a worldwide decrease in the prevalence of dental caries has been observed in recent decades, untreated caries in the permanent dentition remains highly prevalent, affecting about $35 \%$ of the global population. ${ }^{1}$ Costs for direct treatment of dental diseases worldwide are estimated at US\$ 298 billion yearly, corresponding to an average of $4.6 \%$ of global health expenditure. ${ }^{2}$

Since their introduction in the 60 's composite resins have become increasingly popular and currently they are considered universal materials, being the first choice for direct restorations in anterior and posterior teeth. ${ }^{3,4}$ The esthetic characteristics of matching the natural tooth colour, ability to be bonded to tooth tissues, reduced need of sound tooth removal, 
and the low cost compared to indirect materials are some of the reasons for the great popularity of composite resins. ${ }^{5}$

Over the years, several studies have evaluated the clinical longevity of posterior and anterior composite restorations. Systematic reviews have shown that composite restorations might have good clinical performance, with annual failure rates (AFRs) varying from $1 \%$ to $4 \%, 5,6,7,8,9$ However, replacement of restorations is still very frequent in public and private practices, consuming a significant amount of clinical time and imposing high financial costs for health systems. At the end of the last century, it was estimated that $£ 173$ million of annual cost of England's national health care was only for replacing dental restorations..$^{10}$

Clinical studies evaluating the longevity of restorations usually aim to determine the risk factors and reasons for failures of restorations. Whilst the main reasons for failure in posterior restorations are dental caries and fractures of tooth and/or restoration, in anterior teeth the esthetics factor play an important role in the choice of dentist and patient desire for new interventions. ${ }^{6}$ In addition, the decision-making process on how to deal with a restoration with large or small defects can vary widely among dentists with different training status or background. The decision after clinically assessing an old restoration can vary from doing nothing, repairing or replacing. ${ }^{11}$ The decision-making process is probably the most determinant factor for the longevity of restorations. In the past, most studies would concentrate on the clinical performance of different composite materials. Since the current restorative composites seem not to be the problem anymore ${ }^{5}$, more recent literature indicates that other factors have a predominant effect on the clinical performance of composite restorations..$^{12}$ These may include factors related to the patient (age, caries and occlusal stress risk, socioeconomic status) and the professional (age, gender, clinical experience). Establishing risk factors and indicating their main reasons might facilitate dentists' restorative treatment decisions, and consequently increase restorative longevity and reduce costs.

The present manuscript aims to present an overview of the recent literature regarding the clinical performance of direct composite restorations in anterior and posterior teeth and discuss the main factors affecting longevity.

\section{Current evidence on longevity}

In the last five years, systematic reviews have focused on the longevity of composite restorations, assessing AFRs or survival rates. The results of these recent studies were summarized in Table. It is possible to observe that all systematic reviews, even with different search strategies and presenting or not meta-analysis, showed similar results for longevity of direct composite resin restorations, with AFR up to $5 \%$ depending if anterior or posterior teeth were considered and on different times of follow-up.

\section{Aspects influencing longevity}

\section{Patients' related factors}

Although many times neglected in restorative survival analysis, patient-related factors play an important role on the longevity of restorations. Studies have indicated the inclusion of patient factors in the analysis in order to assist with the process of basing clinical decision making on outcomes that are more predictable, and also for patient awareness. Establishing the effect of patients and their related variables is not easy in clinical studies. While age, decayed, missing, and filled teeth index, and socioeconomic status are straightforward variables that can be easily collected, caries risk and parafunctional habits, for instance, are complex processes involving several signs and symptoms, increasing the challenge of choosing the best collection method and criteria to be applied. Since each patient related factors hold its own particularities, the following discussion is presented in topics.

\section{Dental caries}

Caries risk of patients has been shown to significantly influence the longevity of restorations. Studies have been demonstrated that for individuals classified as having high caries risk, the hazard ratio for failure of posterior composite restorations ranged from 2.45 to 4.40 compared with low-risk patients. ${ }^{20,21}$ An increased risk of restoration failure was also observed in direct posterior restorations placed in children which have presented a high DMFT inde $x^{22}$ which for that young age is likely a determinant for caries risk. Among studies on survival of anterior restorations, none 
Table. Systematic reviews on longevity of direct composite restorations published in the last 5 years.

\begin{tabular}{|c|c|c|}
\hline Study & Tooth type & AFR / Survival proportion (SP) \\
\hline Ahmed \& Murbay ${ }^{13}$ & Anterior* & SP: $>90 \%(2.5 \mathrm{yrs}) ; 50 \%$ (5 yrs) \\
\hline Demarco et al. ${ }^{6}$ & Anterior & $\begin{array}{l}\text { AFR: } 0 \text { to } 4.1 \% \\
\text { SP: } 53.4 \text { to } 100 \%\end{array}$ \\
\hline Heintze et al. ${ }^{14}$ & Anterior & $\begin{array}{l}\text { SP: } 95 \%(10 \text { yrs - Class III) } \\
\text { 90\% (10 yrs - Class IV) }\end{array}$ \\
\hline Afrashtehfar et al. ${ }^{15}$ & Posterior** & SP: $89.7 \%$ (3 yrs); $92.4 \%$ (5 yrs) \\
\hline Afrashtehfar et al. ${ }^{16}$ & Posterior & SP: $50 \%$ to $100 \%$ \\
\hline Angeletaki et al. ${ }^{17}$ & Posterior & SP: $83.2 \%$ (5 yrs) \\
\hline Ástvaldsdóttir et al. ${ }^{18}$ & Posterior & SP: $91 \%$ (5 yrs); $86 \%$ (12 yrs) \\
\hline Demarco et al. ${ }^{5}$ & Posterior & AFR: $1 \%$ to $3 \%$ \\
\hline Heintze and Rousson ${ }^{7}$ & Posterior & SP: $90 \%$ (10 yrs) \\
\hline Moraschini et al. ${ }^{19}$ & Posterior & AFR: $3.2 \%$ \\
\hline Opdam et al. ${ }^{9}$ & Posterior & AFR: $1.8 \%$ (5 yrs); $2.4 \%$ (10 yrs) \\
\hline Sande et al. ${ }^{12}$ & Posterior & $\begin{array}{c}\text { AFR: } 1.7 \% \text { to } 5.2 \% \\
\text { SP: } 72 \% \text { to } 95 \%\end{array}$ \\
\hline
\end{tabular}

${ }^{*}$ Tooth wear; ${ }^{* *}$ Endodontically-treated teeth

have investigated this influence, probably motivated by a low incidence of caries found in this region. A recent systematic review on survival of anterior composite restoration have shown a low number of failed restoration due to secondary caries. ${ }^{6}$ However, patients with caries in anterior teeth are typically high-risk patients, thus we can expect on this kind of patient an increased risk for failure of restorations.

Although studies have tried to confirm this evidence, there is still a lack of standardization on the establishment of caries risk profile. Due to multifactorial characteristics of caries disease, several risk indicators (variables) should be collected for a correct identification of graded risk status and future caries prediction, guiding preventive and treatment strategies at the individual level. However, on the investigation of restoration longevity, the use of simplified measures may provide a good estimate of the disease activity when the restoration is placed and in follow-up evaluations. For caries risk, the presence of active caries lesions is still the best predictor for future caries activity.

\section{Bruxism/parafunctional habits}

The excessive habit of grinding and clenching, easily observed on bruxism patients, can heavily affect sound tooth structures over time, resulting in tooth wear and tooth fractures. The same mechanism seems to be associated with an increased risk for restoration fractures. In a retrospective study on survival of composite posterior restoration it was observed that individuals classified as high "occlusal-stress" showed a three times higher risk for failure of restorations than individuals classified as low "occlusal-stress". For the assessment of these conditions, authors took into account objective clinical evaluation (tooth wear signs) along with subjective self-reported parafunctional habits by means of a questionnaire. ${ }^{21}$ The negative effect of bruxism or parafunctional habits on posterior restoration survival was observed in other types of restorative material such as amalgam ${ }^{23}$ and partial-crown ceramic restorations. ${ }^{24}$ In a clinical study on the restoration of severe tooth wear, mainly including bruxing patients, the predominant restoration failure was fracture..$^{25}$ In a study that evaluated build-up restorations placed in worn anterior teeth, fracture of tooth or restoration $(61 \%)$ and restoration loss (30\%) were the most observed reasons of failed restorations, while caries represented only $4 \%$ of failures. ${ }^{23}$

\section{Age}

Several studies have found a significant influence of age group on survival of restorations, and this variable is probably related to other factors. As active caries is the most dominant reason for making a dental restoration in children, those restorations are likely to be in a high risk environment with consequences on longevity. ${ }^{26,27}$ In a study investigating the survival of 
class I and II composite restorations in a large sample of children/adolescents, the age group variable influenced the results, with adolescents (12-19 years) showing a hazard ratio of 0.43 compared with younger children (5-11 years). ${ }^{28}$ The authors discussed that findings could be related to differences in caries risk and the more difficult cooperation of younger children during treatment procedures. For Class II restorations, placed in a practice based study, the higher failure in younger age was also found together with a higher failure risk in older patients..$^{29}$ For the elderly, this might be explained by the presence of more complex restorative work and increased caries risk due to medical complications and decreased oral self care. Therefore, although age may present a significant effect, polarized for the very young and more mature patients, the analysis of the contribution of age on restoration survival, should not be considered as an isolated factor.

\section{Socioeconomic status}

Although still under investigation on survival analysis of restorations, socioeconomic status of patients has been shown to affect the longevity of restorations, ${ }^{30}$ probably motivated by the effect of socioeconomic determinants on dental caries. One of the few studies that investigated this association has shown, in a birth cohort study, that an unfavorable socioeconomic trajectory during life course is associated to a higher presence of subsequent unsatisfactory restorations. ${ }^{30}$ Authors concluded that people who had always lived in the poorest stratus of population had more restoration failures than those who lived in the richest layer. Similar results were observed for posterior restorations placed in a Dutch practice based research. Restorations placed in clinics localized in more deprived areas have shown an increased annual failure rate $(5.6 \%)$ compared to areas considered as a medium (4.2\%) and high (5.1\%) socioeconomic status. ${ }^{31}$

On the other hand, as most of studies on survival of restoration are performed in specialized private practices or highly controlled clinical trials, a more favorable environment may be displayed because patients with a higher socioeconomic background usually attend these facilities, ${ }^{32,33}$ especially considering countries where the dental health system does not rely on public coverage. ${ }^{34}$

\section{Esthetic demand}

Whereas secondary caries is considered together with fracture as the major reason for failure in posterior composite restorations, ${ }^{5,9}$ the aesthetics demands of patients and dentists seem to be a predominant reason for reinterventions on anterior composite restorations. Results of a recent systematic review on anterior restorations shows a larger number of failures related to aesthetics reasons in studies evaluating veneers, diastema closing and reanatonomization teeth as restorations. ${ }^{6}$ High demand for esthetic perfectionism is likely to result in more restorations being replaced for esthetic reasons.

\section{Operator}

Regardless of the dental literature evidence on the longevity of restorations and on the reported causes of failures, probably the "operator factor", which is seldom studied, plays the main role in the whole longevity process. Usually this "operator factor" is explained solely on the basis of the operator training level and accuracy of working, but the decision-making process may well be an underestimated factor and is far more complex and related with co-variables such as the type of practice, reimbursement system, patients' views and opinions, and cultural aspects. In any dental conference or workshop it is possible to see the huge differences among dentists in decisions about how and when to intervene on an existing restoration. These differences are common even among dentists with similar background training, and differences in type of intervention recommended are also common. In practice based studies, differences in restoration longevity among dentists are considerably with more than 2 times higher risk for restoration failure among different practices. ${ }^{29,31}$

Moreover, dental schools usually do not teach how a composite resin restoration changes over time in the intra-oral environment, and how the "natural" degradation process evolves. Several restorative dentistry courses and continuous education activities are focused on the replacement of "ugly" old restoration with small and clinically insignificant defects by brand new and sophisticated "state of art" restorations. This causes in the dental students minds an impression that a good restoration should always be perfect, and look as brand new. Also criteria that are 
used for assessing the quality of dental restorations $\mathrm{s}^{35,36}$ that are designed for identifying already small imperfections are not very helpful, as they may identify many restorations as grade 'charlie' (= soon to be replaced) that could still function without problems in the oral environment for years to come.

In this context, usually small signs of marginal degradation or marginal staining are commonly mistaken as secondary caries lesions. In fact, very few time is dedicated on teaching how to correctly diagnose secondary caries, and this explains the large lack of consensus among dentists about what is secondary caries. Real secondary caries in need for intervention are new caries lesions adjacent to restorations, with clear signs of cavitation and activity. Even then, incipient secondary caries lesions might best be treated by prevention instead of replacing restorations. Moreover, when an operative intervention is required, a repair seems to be the best option. As criteria for repair or replacement of restorations are not clear among dentists, a recent consensus paper has brought this to attention and advised a shift from ' in doubt, take it out' towards ' as a last resort take it out' after considering monitoring, refurbishment, and repair as the better options. ${ }^{11}$

All these factors and lack of consensus explain why the decision-making process on judging restorations is so complex and why the outcome of clinical evaluation of existing restorations increases the risk for replacement of old restorations when the patient change dentists. ${ }^{37}$

\section{Restorative material and technique}

It took some time before resin-based composites were considered as appropriate materials for restoring both anterior and posterior teeth. Poor wear resistance and mechanical strength were shortcomings of the first generations of dental composites, in an era when silver amalgam was the gold standard material for posterior restorations. With time, advances in resin phase composition (e.g. monomers with higher molecular weight and lower polymerization shrinkage) and inorganic filler particles (e.g. higher loading, lower size, and distinct morphologies) led to significant improvements in material properties. Current composites also have higher color stability and better optical properties. In this scenario, problems related to the selection of appropriate restorative composite for placing restorations is a minor problem nowadays. This does not mean that professional preferences and handling conditions, for instance, are insignificant; rather, it indicates that selection of restorative composites currently is not based on clinical performance, since the composite effect is only one of the many aspects influencing the clinical performance. In a systematic review evaluating the performance of posterior composites, the type of composite material was not identified as a significant risk factor for survival, as other factors were, like the patients caries risk and the number of surfaces.

In a scenario where the failures due to inadequate selection of materials are minor, the restorative technique and other clinical conditions can be of more importance. The literature shows that increased cavity size (number of walls involved), ${ }^{9}$ tooth position (posterior teeth have to withstand higher mechanical loadings), ${ }^{9}$ use of a thick layer of glass-ionomer cavity lining, ${ }^{9}$ presence of endodontic treatment, ${ }^{29}$ absence of peripheral enamel approximally ${ }^{38}$ are all factors that might negatively affect clinical longevity. In contrast, often mentioned factors such as enamel beveling, use of rubber dam isolation, and use of direct vs. indirect composite have not been shown to be related to clinical performance. In many situations, such as in the selection of the bonding system, a material-dependent effect is also observed to interfere with clinical performance. ${ }^{39}$

\section{Main reasons for failure}

\section{Secondary caries}

As stated earlier, the two main failure causes reported in the literature are secondary caries and fractures. Considering this high prevalence of secondary caries, the first point that calls for attention is that this prevalence remains more or less the same in the last decades compared to the prevalence of primary caries, which has decreased worldwide. This is interesting and somewhat inexplicable, especially considering that the factors that cause demineralization, which are related to an individual behaviour misbalance on sugar consumption associated with local biofilm accumulation, are exactly the same for primary and secondary caries. Therefore, it can be hypothesized that several clinical cases that are judged as secondary caries are probably 
only marginal defects or marginal staining. In fact, it is pretty common to see dentists reaching a diagnosis of secondary caries in need for intervention looking at non active caries lesions or stained margins. The present trend in cariology that in order to prevent pulp exposure, deeper caries lesions should not be excavated vigorously and caries detection dyes should not be used anymore, will result in more areas of cavity walls with some remaining (residual) caries that will be harmless as the caries process will be stopped by the seal of the restoration. However, as arrested caries may become darker overtime, dentists should be aware that these darker areas around composite restorations are not likely signs of active carious lesions but rather signs of (harmless) arrested caries.

The characteristics of activity for secondary caries are the same as for primary caries. An active caries lesion in enamel is associated with a plaque retentive regions, presents porosity, with a rough and whitish surface. When due to cavitation the dentin is involved, an active caries lesion is humid, soft and brownish tissue, not resistant to mechanical removal with non-rotatory instruments. Therefore, clinicians should act only if signs of activity (progression) are seeing in caries adjacent to restorations, and in most cases the action should be non-operatory (when the lesion is restricted to enamel, for instance). When operatory intervention is needed, repair as an alternative to replacement should be considered.

\section{Fractures}

Short and long term clinical studies on the performance of resin composite restoration $\mathrm{s}^{5,69,18}$ have shown that restoration or tooth/restoration fractures are one of the main reasons for failure in either anterior or posterior teeth. Short-term fractures are usually of minor extension and might be an indicative of technical restorative issues or poor material handling. In contrast, long-term failures are related to fatigue of the restorative complex, involving both the tooth structure and restorative. In the oral environment, the restorations are constantly subjected to physical and chemical challenges. Chemical challenges include those from the humid environment, biofilm accumulation, erosive attacks, and dietary products. Physical challenges involve abrasion and the cyclic mechanical stimulation from chewing. Should the patient have parafunctional habits, the loading imposed to the restorative complex will be much increased. In that scenario and considering all physical-chemical challenges the restorative complex has to withstand, no restoration should be expected to last forever. The grater the cavity size and the composite volume, the greater will be the effects of fatigue in the long term. Although it is a common belief, no clinical study to date have shown that indirect composite restorations might be more resistant to fracture that direct composites. Fractures should be dealt clinically as a common shortcoming of restorative treatments.

\section{Esthetics}

Failures due to aesthetic reasons are rarely reported in studies on posterior restorations. When anterior teeth are considered, importance of aesthetics dramatically increases. ${ }^{32}$ Generally, defects in larger restorations related to color mismatch and surface or marginal staining are easily perceived by patients, influencing negatively their perception. Restorations in anterior teeth also are frequently placed due to an aesthetic demand, increasing patients' expectancy. The literature has shown that the increase in socioeconomic status elevates the search for treatments to improve dental aesthetics. ${ }^{40}$ In this way, the demand for replacement or repair of anterior composite restorations tends to be higher in private dental offices. It is important to highlight that patients' opinion should be always taken in consideration in treatment planning. However, dentists must be careful to avoid both inductions on patient opinion regarding aesthetics and be inducted by patient requirements which can lead to more invasive treatments such as replacements or repairs in cases that can be solved with a simple new polishing of restoration.

\section{Future perspectives}

Future studies should be focused on less technique sensitive materials, since properties of current composites seem to be adequate to provide long lasting results. Less technique sensitive procedures and materials might reduce the errors produced by the operators. Considering that dental caries and occlusal disturbances may significantly reduce the longevity of composite restorations, studies should be developed in 
in real life situations like general practices or population based to identify all possible risk factors on material, tooth, patient and practice level. In addition, the costeffectiveness of esthetic treatments using different esthetic restoratives (e.g. composite vs. ceramic) should be considered in future studies. Finally, there are still questions to be answered in clinical studies, including the effect of mechanical loading on the development of caries adjacent to composite restorations, the impact of suboptimal photoactivation procedures and failed bonded interfaces on composite longevity, and if there is still room for materials improvement using the methacrylate technology.

\section{Conclusion}

In conclusion:

Composite restorations have been shown to perform favourably in posterior teeth and anterior teeth

\section{References}

1. Marcenes W, Kassebaum NJ, Bernabé E, Flaxman A, Naghavi M, Lopez A et al. Global burden of oral conditions in 1990-2010: a systematic analysis. J Dent Res. 2013;92(7):592-7. https://doi.org/10.1177/0022034513490168

2. Listl S, Galloway J, Mossey PA, Marcenes W. Global economic impact of dental diseases. J Dent Res. 2015;94(10):1355-61. https://doi.org/10.1177/0022034515602879

3. Correa MB, Peres MA, Peres KG, Horta BL, Barros AD, Demarco FF. Amalgam or composite resin? Factors influencing the choice of restorative material. J Dent. 2012;40(9):703-10. https://doi.org/10.1016/i.jdent.2012.04.020

4. Nascimento GG, Correa MB, Opdam N, Demarco FF. Do clinical experience time and postgraduate training influence the choice of materials for posterior restorations? Results of a survey with Brazilian general dentists. Braz Dent J. 2013;24(6):642-6. https://doi.org/10.1590/0103-6440201302361

5. Demarco FF, Corrêa MB, Cenci MS, Moraes RR, Opdam NJM. Longevity of posterior composite restorations: not only a matter of materials. Dent Mater. 2012;28(1):87-101. https://doi.org/10.1016/i.dental.2011.09.003

6. Demarco FF, Collares K, Coelho-de-Souza FH, Correa MB, Cenci MS, Moraes RR et al. Anterior composite restorations: a systematic review on long-term survival and reasons for failure. Dent Mater. 2015;31(10):1214-24. https://doi.org/10.1016/j.dental.2015.07.005
Failure behavior in anterior restorations is different from posterior teeth, with less secondary caries present and more restorations being replaced for esthetic appearance.

The longevity of composite restorations might be impaired by the lack of criteria for intervention on existing restorations, and by misdiagnosis of secondary caries.

Several risk factors on practice/operator, patient, and tooth/restoration level influence the longevity of dental restorations

Specific patient related risk factors are difficult to assess clinically and there is a lack of standardization.

Dentistry should place more effort on training dentists in how to deal with imperfect restorations.

Clinical trials should have rational control groups, include confounders as patient risk factors in the data and analysis and should use outcome parameters relevant for profession and patients.
7. Heintze SD, Rousson V. Clinical effectiveness of direct class II restorations: a meta-analysis. J Adhes Dent. 2012;14(5):407-31. https://doi.org/10.3290/i.jad.a28390

8. Manhart J, Chen H, Hamm G, Hickel R. Buonocore Memorial Lecture. Review of the clinical survival of direct and indirect restorations in posterior teeth of the permanent dentition. Oper Dent. 2004;29(5):481-508

9. Opdam NJ, van de Sande FH, Bronkhorst E, Cenci MS, Bottenberg P, Pallesen $U$ et al. Longevity of posterior composite restorations: a systematic review and meta-analysis. J Dent Res. 2014;93(10):943-9. https://doi.org/10.1177/0022034514544217

10. Dissimination NCfRa. Dental restoration: what type of filling? Eff Health Care. 1999;5(2):1-12.

11. Wilson N, Lynch CD, Brunton PA, Hickel R, Meyer-Lueckel H, Gurgan $\mathrm{S}$ et al. Criteria for the replacement of restorations: Academy of Operative Dentistry European Section. Oper Dent. 2016;41 S7:S48-57. https://doi.org/10.2341/15-058-O

12. Sande FH, Collares K, Correa MB, Cenci MS, Demarco FF, Opdam N. Restoration survival: revisiting patients' risk factors through a systematic literature review. Oper Dent. 2016;41(S7):S7-S26. https://doi.org/10.2341/15-120-LIT

13. Ahmed KE, Murbay S. Survival rates of anterior composites in managing tooth wear: systematic review. J Oral Rehabil. 2016;43(2):145-53. https://doi.org/10.1111/joor.12360 
14. Heintze SD, Rousson V, Hickel R. Clinical effectiveness of direct anterior restorations: a meta-analysis. Dent Mater. 2015;31(5):481-95. https://doi.org/10.1016/i.dental.2015.01.015

15. Afrashtehfar KI, Ahmadi M, Emami E, Abi-Nader S, Tamimi F. Failure of single-unit restorations on root filled posterior teeth: a systematic review. Int Endod J. 2017. https://doi.org/10.1111/iej.12723

16. Afrashtehfar KI, Emami E, Ahmadi M, Eilayyan O, Abi-Nader S, Tamimi F. Failure rate of single-unit restorations on posterior vital teeth:a systematic review. J Prosthet Dent. 2017;117(3):345-53.e8. https://doi.org/10.1016/i.prosdent.2016.08.003

17. Angeletaki F, Gkogkos A, Papazoglou E, Kloukos D. Direct versus indirect inlay/onlay composite restorations in posterior teeth: a systematic review and meta-analysis. J Dent. 2016;53:12-21.

18. Ástvaldsdóttir Á, Dagerhamn J, van Dijken JW, Naimi-Akbar A, Sandborgh-Englund G, Tranæus $S$ et al. Longevity of posterior resin composite restorations in adults: a systematic review. J Dent. 2015;43(8):934-54. https://doi.org/10.1016/i.jdent.2016.07.011

19. Moraschini V, Fai CK, Alto RM, Santos GO. Amalgam and resin composite longevity of posterior restorations: a systematic review and meta-analysis. J Dent. 2015;43(9):1043-50. https://doi.org/10.1016/i.jdent.2015.06.005

20. Opdam NJ, Bronkhorst EM, Roeters JM, Loomans BA. A retrospective clinical study on longevity of posterior composite and amalgam restorations. Dent Mater. 2007;23(1):2-8. https://doi.org/10.1016/j.dental.2005.11.036

21. Sande FH, Opdam NJ, Rodolpho PA, Correa MB, Demarco FF, Cenci MS. Patient risk factors' influence on survival of posterior composites. J Dent Res. 2013;92(7 Suppl):78-83S. https://doi.org/10.1177/0022034513484337

22. Trachtenberg F, Maserejian NN, Tavares M, Soncini JA, Hayes $C$. Extent of tooth decay in the mouth and increased need for replacement of dental restorations: the New England Children's Amalgam Trial. Pediatr Dent. 2008;30(5):388-92.

23. Smales RJ, Berekally TL. Long-term survival of direct and indirect restorations placed for the treatment of advanced tooth wear. Eur J Prosthodont Restor Dent. 2007;15(1):2-6.

24. Dijken JW, Hasselrot L. A prospective 15-year evaluation of extensive dentin-enamel-bonded pressed ceramic coverages. Dent Mater. 2010;26(9):929-39. https://doi.org/10.1016/i.dental.2010.05.008

25. Hamburger JT, Opdam NJ, Bronkhorst EM, Huysmans MC. Indirect restorations for severe tooth wear: fracture risk and layer thickness. J Dent. 2014;42(4):413-8. https://doi.org/10.1016/j.jdent.2013.10.003

26. Bernardo M, Luis $H$, Martin MD, Leroux BG, Rue T, Leitão J et al. Survival and reasons for failure of amalgam versus composite posterior restorations placed in a randomized clinical trial. J Am Dent Assoc. 2007;138(6):775-83. https://doi.org/10.14219/jada.archive.2007.0265

27. Soncini JA, Maserejian NN, Trachtenberg F, Tavares $M$, Hayes $C$. The longevity of amalgam versus compomer/composite restorations in posterior primary and permanent teeth: findings from the New England Children's
Amalgam Trial. J Am Dent Ass. 2007;138(6):763-72.

https://doi.org/10.14219/jada.archive.2007.0264

28. Pallesen U, van Dijken JWV, Halken J, Hallonsten AL, Höigaard R. A prospective 8-year follow-up of posterior resin composite restorations in permanent teeth of children and adolescents in Public Dental Health Service: reasons for replacement. Clin Oral Investig. 2014;18(3):819-27. https://doi.org/10.1007/s00784-013-1052-x

29. Laske M, Opdam NJ, Bronkhorst EM, Braspenning JC, Huysmans MC. Ten-year survival of class II restorations placed by general practitioners. JDR Clin Trans Res. 2016;1(3):292-9.

30. Correa MB, Peres MA, Peres KG, Horta BL, Barros AJ, Demarco FF. Do socioeconomic determinants affect the quality of posterior dental restorations? A multilevel approach. J Dent. 2013;41(11):960-7. https://doi.org/10.1016/i.jdent.2013.02.010

31. Laske M, Opdam NJ, Bronkhorst EM, Braspenning JC, Huysmans MC. Longevity of direct restorations in Dutch dental practices: descriptive study out of a practice based research network. J Dent. 2016;46:12-7. https://doi.org/10.1016/i.jdent.2016.01.002

32. Baldissera RA, Corrêa MB, Schuch HS, Collares K, Nascimento GG, Jardim PS et al. Are there universal restorative composites for anterior and posterior teeth? J Dent. 2013;41(11):1027-35. https://doi.org/10.1016/i.jdent.2013.08.016

33. Rodolpho PAR, Donassollo TA, Cenci MS, Loguércio AD, Moraes RR, Bronkhorst EM et al. 22-Year clinical evaluation of the performance of two posterior composites with different filler characteristics. Dent Mater. 2011;27(10):955-63. https://doi.org/10.1016/i.dental.2011.06.001

34. Palència L, Espelt A, Cornejo-Ovalle M, Borrell C. Socioeconomic inequalities in the use of dental care services in Europe: what is the role of public coverage? Community Dent Oral Epidemiol. 2014;42(2):97-105. https://doi.org/10.1111/cdoe.12056

35. Hickel R, Peschke A, Tyas M, Miör I, Bayne S, Peters $M$ et al. FDI World Dental Federation - clinical criteria for the evaluation of direct and indirect restorations: update and clinical examples. J Adhes Dent. 2010;12(4):259-72. https://doi.org/10.1007/s00784-010-0432-8

36. Ryge G, Snyder M. Evaluating the clinical quality of restorations. J Am Dent Ass. 1973;87(2):369-77.

37. Burke FJ, Lucarotti PS. How long do direct restorations placed within the general dental services in England and Wales survive? $\mathrm{Br}$ Dent J. 2009;206(1):E2. https://doi.org/10.1038/sj.bdj.2008.1042.

38. Kuper NK, Opdam NJ, Ruben JL, de Soet JJ, Cenci MS, Bronkhorst EM et al. Gap size and wall lesion development next to composite. J Dent Res. 2014;93(7 Suppl):108-13S. https://doi.org/10.1177/0022034514534262

39. Collares K, Corrêa MB, Laske M, Kramer E, Reiss B, Moraes RR et al. A practice-based research network on the survival of ceramic inlay/onlay restorations. Dent Mater. 2016;32(5):687-94. https://doi.org/10.1016/j.dental.2016.02.006

40. Špali S, Katić V, Vidaković R, Šlaj M, Šlaj M. History of orthodontic treatment, treatment needs and influencing factors in adolescents in Croatia. Cent Eur J Public Health. 2016;24(2):123-7. https://doi.org/10.21101/cejph.a4117 